\title{
Variation of radiation length due to LPM effect
}

\author{
V. N. Baier and V. M. Katkov \\ Budker Institute of Nuclear Physics \\ 630090 Novosibirsk, Russia
}

November 12, 2018

\begin{abstract}
The photon emission intensity spectrum is calculated taking into account influence of multiple scattering (the LPM effect) under conditions of recent CERN SPS experiment. It is shown that the theory quite satisfactory describes data. The integral characteristics: the radiation length and total probability of photon emission are analyzed under influence of the LPM effect, and the asymptotic expansions of these characteristics are derived.
\end{abstract}




\section{Introduction}

When a charged particle is moving in a medium it scatters on atoms. With probability $\sim \alpha$ this scattering is accompanied by a radiation. At high energy the radiation process occurs over a rather long distance, known as the formation length $l_{c}$ (see e.g. [1]):

$$
l_{c}=\frac{l_{0}}{1+\gamma^{2} \vartheta_{c}^{2}}, \quad l_{0}=\frac{2 \varepsilon \varepsilon^{\prime}}{m^{2} \omega},
$$

where $\omega$ is the energy of emitted photon, $\varepsilon(m)$ is the energy (the mass) of a particle, $\varepsilon^{\prime}=\varepsilon-\omega, \vartheta_{c}$ is the characteristic angle of photon emission, the system $\hbar=c=1$ is used.

Landau and Pomeranchuk were the first who showed that if the formation length of bremsstrahlung becomes comparable to the distance over which the multiple scattering becomes important, the bremsstrahlung will be suppressed [2]. Migdal [3] developed the quantitative theory of this phenomenon.

A new interest to the theory of the LPM effect is connected with a very successful series of experiments performed at SLAC 4]. In these experiments the cross section of the bremsstrahlung of soft photons with energy from $200 \mathrm{keV}$ to $500 \mathrm{MeV}$ from electrons with energy $8 \mathrm{GeV}$ and $25 \mathrm{GeV}$ is measured with an accuracy of the order of a few percent. Both LPM and dielectric suppression are observed and investigated. The logarithmic binning of photon spectrum was used with 25 bins per decade. These experiments were the challenge for the theory since in all the mentioned papers calculations are performed to logarithmic accuracy which is not enough for description of the new experiment. The contribution of the Coulomb corrections (at least for heavy elements) is larger than experimental errors and these corrections should be taken into account.

Recently new study of LPM effect at higher energies of electrons $(\varepsilon=149,207$ and $287 \mathrm{GeV}$ ), where the effect has influence upon much wider part of spectrum comparing with $\varepsilon=25 \mathrm{GeV}$, was performed in the $\mathrm{H} 2$ beam line of the CERN SPS [5], [6]. The logarithmic binning of photon spectrum was used with 25 bins per decade as at SLAC.

We developed the new approach to the theory of the Landau-PomeranchukMigdal (LPM) effect [7]. In this paper the cross section of the bremsstrahlung process in the photon energies region where the influence of the LPM is very strong was calculated with a term $\propto 1 / L$, where $L$ is characteristic logarithm of the problem, and with the Coulomb corrections taken into account. In the photon energy region, where the LPM effect is "turned off", the obtained cross section gives the exact Bethe-Maximon cross section (within power accuracy and with the Coulomb corrections). This important feature was absent in the previous calculations. The LPM effect in a thin target were the interference effects and boundary photon emission is of special interest was analyzed in [8]. The probability of multiphoton emission is enhanced at high energy 9]. Correspondingly this effect is very important for comparison of theory prediction and data. The influence of LPM effect on integral characteristics of bremsstrahlung was considered in [10]. The other approaches to the LPM effect theory see e.g. in [1], 12], the recent review is given in [13].

In Sec. 2 the theory predictions are compared with the recent CERN SPS data [5], 6]. It is shown that the theory quite satisfactory describes data. In Sec.3 
the variation of the radiation length due to multiple scattering is discussed. In the region where the LPM effect is weak $\left(\varepsilon \ll \varepsilon_{e}\right)$ the asymptotic expressions for the radiation length and the photon emission probability are derived taking into account both decomposition over $\varepsilon / \varepsilon_{e}$ and over $1 / L_{1}, L_{1}$ is the characteristic logarithm of the problem. Appendices $\mathrm{A}$ and $\mathrm{B}$ contains details of derivation for radiation length and Appendix $\mathrm{C}$ for the photon emission probability. In Appendix D the structure of series over $\varepsilon / \varepsilon_{e}$ and over $1 / L_{1}$ is analyzed for $\varepsilon \ll \varepsilon_{e}$.

\section{Influence of the multiple scattering on the bremsstrahlung spectrum}

The spectral radiation intensity obtained in [7], Eq.(2.39) (see also Sec.3 in [10]) is valid for any energy and has the form

$$
d I=\omega d W=\frac{\alpha m^{2} x d x}{2 \pi(1-x)} \operatorname{Im}\left[\Phi(\nu)-\frac{1}{2 L_{c}} F(\nu)\right], \quad x=\frac{\omega}{\varepsilon},
$$

where

$$
\begin{aligned}
& \Phi(\nu)=\int_{0}^{\infty} d z e^{-i t}\left[r_{1}\left(\frac{1}{\sinh z}-\frac{1}{z}\right)-i \nu r_{2}\left(\frac{1}{\sinh ^{2} z}-\frac{1}{z^{2}}\right)\right] \\
& =r_{1}\left(\ln p-\psi\left(p+\frac{1}{2}\right)\right)+r_{2}\left(\psi(p)-\ln p+\frac{1}{2 p}\right) \\
& F(\nu)=\int_{0}^{\infty} \frac{d z e^{-i t}}{\sinh ^{2} z}\left[r_{1} f_{1}(z)-2 i r_{2} f_{2}(z)\right], \\
& t=\frac{z}{\nu}, \quad r_{1}=x^{2}, \quad r_{2}=1+(1-x)^{2} .
\end{aligned}
$$

where $z=\nu t, p=i /(2 \nu), \psi(x)$ is the logarithmic derivative of the gamma function. The functions $f_{1}(z)$ and $f_{2}(z)$ are defined by following expressions

$$
\begin{aligned}
& f_{1}(z)=\left(\ln \varrho_{c}^{2}+\ln \frac{\nu}{i}-\ln \sinh z-C\right) g(z)-2 \cosh z G(z), \\
& f_{2}(z)=\frac{\nu}{\sinh z}\left(f_{1}(z)-\frac{g(z)}{2}\right), \quad g(z)=z \cosh z-\sinh z, \\
& G(z)=\int_{0}^{z}(1-y \operatorname{coth} y) d y \\
& =z-\frac{z^{2}}{2}-\frac{\pi^{2}}{12}-z \ln \left(1-e^{-2 z}\right)+\frac{1}{2} \operatorname{Li}_{2}\left(e^{-2 z}\right),
\end{aligned}
$$

here $\mathrm{Li}_{2}(x)$ is the Euler dilogarithm. Use of the last representation of function $G(z)$ simplifies the numerical calculation. The crucial parameter $\nu$ is

$$
\begin{aligned}
& \nu^{2}=i \nu_{0}^{2}, \quad \nu_{0}^{2}=|\nu|^{2} \simeq \nu_{1}^{2}\left(1+\frac{\ln \nu_{1}}{L_{1}} \vartheta\left(\nu_{1}-1\right)\right), \quad \nu_{1}^{2}=\frac{\varepsilon}{\varepsilon_{e}} \frac{1-x}{x}, \\
& \varepsilon_{e}=m\left(8 \pi Z^{2} \alpha^{2} n_{a} \lambda_{c}^{3} L_{1}\right)^{-1}, \quad L_{c} \simeq L_{1}\left(1+\frac{\ln \nu_{1}}{L_{1}} \vartheta\left(\nu_{1}-1\right)\right), \quad L_{1}=\ln \frac{a_{s 2}^{2}}{\lambda_{c}^{2}}, \\
& \frac{a_{s 2}}{\lambda_{c}}=183 Z^{-1 / 3} \mathrm{e}^{-f}, \quad f=f(Z \alpha)=(Z \alpha)^{2} \sum_{k=1}^{\infty} \frac{1}{k\left(k^{2}+(Z \alpha)^{2}\right)},
\end{aligned}
$$


where $Z$ is the charge of the nucleus, $n_{a}$ is the number density of atoms in the medium, $\lambda_{c}=1 / m=(\hbar / m c)$ is the electron Compton wavelength. Here $\varepsilon_{e}$ is a characteristic parameter of medium, starting from this energy the multiple scattering distorts the whole spectrum of bremsstrahlung including its hard part: for iridium $\varepsilon_{e}=2.27 \mathrm{TeV}$, for gold $\varepsilon_{e}=2.6 \mathrm{TeV}$, for tungsten $\varepsilon_{e}=2.73 \mathrm{TeV}$, for tantalum $\varepsilon_{e}=3.18 \mathrm{TeV}$, for lead $\varepsilon_{e}=4.38 \mathrm{TeV}$.

In the case $\varepsilon \ll \varepsilon_{e}$ the LPM effect manifests itself when

$$
\nu_{1}\left(x_{c}\right)=1, \quad x_{c}=\frac{\varepsilon}{\varepsilon_{e}+\varepsilon} \simeq \frac{\varepsilon}{\varepsilon_{e}} .
$$

In this case in the hard part of spectrum $\left(1 \geq x \gg x_{c}, \nu_{1}^{2} \simeq x_{c} / x \ll 1\right)$ one has (see Appendices A and B)

$$
\begin{aligned}
& \frac{d I}{d x}=\frac{\varepsilon}{L_{\text {rad }}^{0}}\left\{x^{2}+\frac{4(1-x)}{3}+\frac{2(1-x)}{9 L_{1}}-\frac{\varepsilon^{2}}{\varepsilon_{e}^{2}} \frac{(1-x)^{2}}{x^{2}}\right. \\
& \left.\times\left[\left(\frac{64}{63}-\frac{15272}{2205} \frac{1}{L_{1}}\right)(1-x)+\left(1-\frac{5017}{1800} \frac{1}{L_{1}}\right) x^{2}\right]\right\}, \\
& \frac{1}{L_{r a d}^{0}}=\frac{2 Z^{2} \alpha^{3} n_{a} L_{1}}{m^{2}}=\frac{\alpha}{4 \pi} \frac{m}{\varepsilon_{e} \lambda_{c}}, \quad \frac{1}{L_{r a d}^{B M}}=\frac{1}{L_{r a d}^{0}}\left(1+\frac{1}{9 L_{1}}\right),
\end{aligned}
$$

here $L_{\text {rad }}^{B M}$ is the Bethe-Maximon radiation length. Note that if neglect here the terms $\propto \varepsilon^{2} / \varepsilon_{e}^{2}$ we obtain the Bethe-Maximon intensity spectrum.

In the recent CERN SPS experiment the iridium target with thickness $l=0.128 \mathrm{~mm}$ $\left(l / L_{\text {rad }}=4.36 \pm 0.10 \%, L_{\text {rad }}\right.$ is the radiation length $)[5]$ and tantalum target 6 ] with thickness $l / L_{\text {rad }}=4.45 \pm 0.10 \%$ were used. Photons with energy $2 \mathrm{GeV}$ $<\omega<\varepsilon$ were detected in a lead glass calorimeter.

Obtained experimental data should be recalculated:

$$
\left(\frac{d \varepsilon}{d \omega}\right)_{e x p}=\frac{l}{L_{r a d}} \frac{1}{k}\left(\frac{d N}{d \ln \omega}\right)_{e x p} .
$$

Because photon energies were histogrammed logarithmically, using 25 bins per decade of energy, one has for the coefficient $k$

$$
\begin{aligned}
& k_{h}=\frac{\omega_{\max }-\omega_{\min }}{\omega_{\min }}=e^{s}-1=0.096, \quad k_{m}=2 \frac{\omega_{\max }-\omega_{\min }}{\omega_{\max }+\omega_{\min }}=2 \frac{e^{s}-1}{e^{s}+1}=0.092, \\
& k_{l}=\frac{\omega_{\max }-\omega_{\min }}{\omega_{\max }}=1-e^{-s}=0.88, \quad s=\ln \frac{\omega_{\max }}{\omega_{\min }}=\frac{\ln 10}{25}=0.092,
\end{aligned}
$$

depending on the normalization point within bin. Here we will use $k_{m}$.

Since only high energy photons $(\omega \geq 2 \mathrm{GeV})$ were measured, no boundary effects were observed. So, only the pure LPM effect was studied. For iridium $\varepsilon_{e}=2.27 \mathrm{TeV}$ and from one has Eq.(2.5) that the characteristic photon energy $\omega_{c}(\varepsilon)$ for which the LPM effect is well manifests itself is $\omega_{c}(287 \mathrm{GeV})=32 \mathrm{GeV}$ and $\omega_{c}(207 \mathrm{GeV})=19 \mathrm{GeV}$, while for tantalum $\varepsilon_{e}=3.18 \mathrm{TeV}$ and $\omega_{c}(287 \mathrm{GeV})=26 \mathrm{GeV}$. The results of calculations for iridium (the initial electron energy $\varepsilon=287 \mathrm{GeV}$ and $\varepsilon=207 \mathrm{GeV}$ ) and for tantalum (the initial electron energy $\varepsilon=287 \mathrm{GeV}$ ) are shown in Figs.1-3. The curve 1 is the Bethe-Maximon intensity spectrum (see e.g.Eq.(2.6) ). The curves 2, 3 are calculated using Eqs. (2.1) and (2.2). The 
curve 2 presents the main term (the function $\Phi(\nu)$ ), the curve 3 presents the correction term (the function $F(\nu)$ ). It should be noted that the prediction of our theory (sum of previous terms, curve 4) in the hard end of spectrum coincide with the Bethe-Maximon curve within the accuracy better than $10^{-3}$. It is seen that given values $\omega_{c}(\varepsilon)$ show the scale where LPM effect becomes essential. For used thickness of target the multi-photon effects are very essential. The reduction factor $f$ in this case it is convenient to calculate using the following general expression (Eq.(3.4) of [9]):

$$
\frac{d \varepsilon}{d \omega}=\omega \frac{d w}{d \omega} f, \quad f=\exp \left[-\int_{\omega}^{\infty} \frac{d w}{d \omega_{1}} d \omega_{1}\right]\left(1+O\left(\omega \frac{d w}{d \omega}\right)\right),
$$

where the main term (with $\Phi(\nu)$ ) of spectrum Eq.(2.1) is substituted. The results obtained are in a good agreement with Eqs.(2.11) and (2.26) of [9]. The reduction factor $f$ in iridium for three used energies is presented in Fig.4. It follows from Fig. 4 that if the electron energy decreases the reduction factor $f$ diminishes as well. The final prediction, with the reduction factor taken into account for used target thicknesses, present the curves $T$. The data are recalculated according with Eqs.(2.7),(2.8) using the coefficient $k_{m}$. In Figs.1-3 one can see that for energy $287 \mathrm{GeV}$ there is quite satisfactory agreement of theory with data for both iridium and tantalum, for energy $207 \mathrm{GeV}$ in iridium the agreement is somewhat less satisfactory.

\section{The LPM radiation length and the average number of emitted photons at $\varepsilon \ll \varepsilon_{e}$}

The local radiation length $L_{\text {rad }}$ is defined by equation

$$
\frac{d \varepsilon}{d t}=-I(\varepsilon)=-\frac{\varepsilon}{L_{\text {rad }}} .
$$

In the absence of LPM effect the value $L_{\text {rad }}$ doesn't dependent on energy and defined by Eq.(2.6). In this case, after averaging Eq.(3.1) with the electron distribution function over energy $f(\varepsilon, t)$ one obtains the closed equation for the mean energy loss which has the solution $\langle\varepsilon(t)\rangle=\varepsilon_{0} \exp \left(-t / L_{\text {rad }}\right)$.

For the first photon emission according with Eq.(3.1) $L_{\text {rad }}=\varepsilon / I(\varepsilon)$. The dependence on the energy of the function $(I(\varepsilon) / \varepsilon) L_{\text {rad }}^{B M}=L_{\text {rad }}^{B M} / L_{\text {rad }}$ for iridium (curve 1) and lead (curve 2) is given in Fig.5. The $I(\varepsilon)$ is the integrated over $x$ Eq.(2.1). The relative value of correction (the term with $F(\nu)$ ) to the main term (with $\Phi(\nu)$ ) depends on energy. It attains the maximum $\sim 5 \%$ near $\varepsilon \sim \varepsilon_{e}$ for both media. So, the value of $L_{\text {rad }}$ for the energy $\varepsilon=3 \mathrm{TeV}$ increases 1.33 times in $\mathrm{Ir}$ and in 1.16 times in $\mathrm{Pb}$, while for the energy $\varepsilon=10 \mathrm{TeV}$ the corresponding figures are 1.58 and 1.38 .

In the region where the LPM effect is weak (see Appendices A and B)

$$
\begin{aligned}
& \frac{1}{L_{\text {rad }}}=\frac{1}{L_{\text {rad }}^{0}}\left\{1+\frac{1}{9 L_{1}}-\frac{4 \pi}{15} \frac{\varepsilon}{\varepsilon_{e}}+\frac{64}{21} \frac{\varepsilon^{2}}{\varepsilon_{e}^{2}}\left(\ln \frac{\varepsilon_{e}}{\varepsilon}-2.040\right)\right. \\
& +\frac{1}{L_{1}}\left[\frac{182 \pi}{225} \frac{\varepsilon}{\varepsilon_{e}}-\frac{15272}{735} \frac{\varepsilon^{2}}{\varepsilon_{e}^{2}}\left(\ln \frac{\varepsilon_{e}}{\varepsilon}-2.577\right)\right] .
\end{aligned}
$$


The first term of expansion over $\varepsilon / \varepsilon_{e}$ (not over $1 / L_{1}$ ) was found in [10].

The accuracy of Eq. (3.2) is defined by a several essentially different factors. The first is the relative value of discarded terms $O\left(\varepsilon^{3} / \varepsilon_{e}^{3}\right)$ in Eqs. (A.13), B.15). The second is the substitution of $L_{c}$ by $L_{1}\left(\varrho_{c}=1\right)$ in Eqs.(2.1) - (2.4) for the whole spectrum, the relative accuracy of this substitution is $O\left(\varepsilon /\left(\varepsilon_{e} L_{1}^{2}\right)\right)$. The third is due to fact that in the initial formula Eq.(2.1) the terms $\propto 1 / L_{c}^{2}$ were rejected. However, as is shown in in Appendix D, in the region $\varepsilon \ll \varepsilon_{e}$ the corrections $\propto 1 / L_{1}^{2}$ are beginning with the terms contained $\varepsilon / \varepsilon_{e}$. So, in this region, the relative accuracy in the formula Eq.(2.1) is also $O\left(\varepsilon /\left(\varepsilon_{e} L_{1}^{2}\right)\right)$.

One can see from Eq.(3.2), that in a decomposition over $\varepsilon / \varepsilon_{e}$ one have to take into account the terms $\propto 1 / L_{1}$ because of relatively large numerical values of the coefficients of decomposition. Moreover, for heavy elements $L_{1} \simeq(6 \div 7)$ taking into account of term $\propto 1 / L_{1}$ results in substantial change of numerical value of coefficients at the given degree of $\varepsilon / \varepsilon_{e}$. Particularly this is important for term containing $\ln \left(\varepsilon_{e} / \varepsilon\right)$. As a result, there is essential compensation inside the coefficients of decomposition. This permit to use the decomposition Eq.(3.2) at relatively large energies. At $\varepsilon / \varepsilon_{e} \leq 1 / 10$ an error in the correction terms doesn't exceed $10 \%$.

As illustration we present the radiation length in iridium $\left(L_{1}=6.9225, \varepsilon_{e}=\right.$ $\left.2.27 \mathrm{TeV}, L_{\text {rad }}^{B M}(\mathrm{Ir})=2.91 \mathrm{~mm}\right)$ :

$$
\frac{1}{L_{r a d}(\mathrm{Ir})}=\frac{1}{L_{\text {rad }}^{B M}(\mathrm{Ir})}\left(1-0.464 \frac{\varepsilon}{\varepsilon_{e}}+0.045 \frac{\varepsilon^{2}}{\varepsilon_{e}^{2}} \ln \frac{\varepsilon_{e}}{\varepsilon}+1.492 \frac{\varepsilon^{2}}{\varepsilon_{e}^{2}}\right) .
$$

It should be noted that after substitution of Eq.(3.3) into Eq.(3.1) and averaging with the distribution function $f(\varepsilon, t)$ the higher moments of $\varepsilon$ appear. Because of this, equation (3.1) ceases to be closed. We will discuss this item elsewhere.

The mean value of photons emitted by electron is also of evident interest. Particularly, at $\omega \ll \omega_{c} \simeq \varepsilon^{2} / \varepsilon_{e}$ this value defines mainly the exponent in Eq.(2.9). At energy where the LPM effect is rather weak $\left(\varepsilon \ll \varepsilon_{e}\right)$ the radiation probability per unit time integrated over whole photon spectrum is (see Appendix C)

$$
\begin{aligned}
& W(\varepsilon)=\frac{d w}{d t}=\frac{\alpha m}{3 \pi \varepsilon_{e} \lambda_{c}}\left[\ln \frac{\varepsilon_{e}}{\varepsilon}+1.959+\frac{2 \pi}{5} \frac{\varepsilon}{\varepsilon_{e}}\right. \\
& \left.+\frac{1}{L_{1}}\left(\frac{1}{6} \ln \frac{\varepsilon_{e}}{\varepsilon}+3.574-\frac{91 \pi}{75} \frac{\varepsilon}{\varepsilon_{e}}\right)\right] .
\end{aligned}
$$

It is seen that allowing for terms $\propto 1 / L_{1}$ changes essentially non-logarithmic terms in Eq.(3.4).

\section{Acknowledgments}

The authors are indebted to the Russian Foundation for Basic Research supported in part this research by Grant 03-02-16154.

We would like to thank U.Uggerhoj for information about experiment and data. 


\section{A Appendix}

We consider here the asymptotic behavior of the total intensity of radiation $I_{0}$ in the region $\varepsilon \ll \varepsilon_{e}$ where the LPM effect is weak and one can put in Eqs.(2.1)-(2.4) $\nu^{2}=i \nu_{1}^{2}, \varrho_{c}=1, L_{c}=L_{1}$. This substitution will manifest itself only starting from

terms of the order $\frac{1}{L_{1}^{2}} \frac{\varepsilon}{\varepsilon_{e}} I_{0}$. Then in expressions for $\Phi(\nu)$ and $F(\nu)$ in Eq.(2.2) we integrate by parts the terms $\propto r_{1}$ and make the substitution of variable $t \rightarrow-i t$. As a result we obtain

$$
I=\frac{\varepsilon}{L_{\text {rad }}^{0}}\left(J_{m}+\frac{1}{L_{1}} J_{v}\right)
$$

where

$$
\begin{aligned}
& J_{m}=2 \operatorname{Re} \int_{0}^{1} d x \int_{0}^{\infty} d t e^{-t}\left[x^{2} \varphi_{1}(z)-2(1-x) \varphi_{2}(z)\right] \\
& \varphi_{1}(z)=\frac{1}{\cosh z+1}, \quad \varphi_{2}(z)=\frac{1}{\sinh ^{2} z}-\frac{1}{z^{2}}
\end{aligned}
$$

and

$$
\begin{aligned}
& J_{v}=-\operatorname{Re} \int_{0}^{1} d x \int_{0}^{\infty} d t e^{-t}\left[x^{2} l_{1}(z, t)+4(1-x) l_{2}(z, t)\right] \\
& l_{1,2}=a_{1,2}(z)(\ln t+C)+b_{1,2}(z), a_{1}(z)=\varphi_{1}(z)-\frac{z}{\sinh z}, a_{2}(z)=\frac{z \operatorname{coth} z-1}{\sinh ^{2} z}, \\
& b_{1}(z)=a_{1}(z) \ln \frac{\sinh z}{z}+\frac{1}{\cosh z+1}\left(1-z \operatorname{coth} z+\frac{2}{\sinh z} G(z)\right), \\
& b_{2}(z)=a_{2}(z) \ln \frac{\sinh z}{z}+\frac{1}{2 \sinh ^{2} z}(4 G(z) \operatorname{coth} z+z \operatorname{coth} z-1),
\end{aligned}
$$

here the function $G(z)$ is defined in Eq.(2.3) and

$$
\beta^{2}=i \frac{\varepsilon_{e}}{\varepsilon}, \quad z=\frac{t}{\beta y}, \quad y=\sqrt{\frac{x}{1-x}} .
$$

In Eq. (A.2) one can expand the function $\varphi_{1}(z)$ up to terms $\propto z^{4}$. We find

$$
J_{m 1} \simeq 2 \int_{0}^{1} d x x^{2} \int_{0}^{\infty} d t e^{-t}\left(\varphi_{1}(0)+\frac{\varphi_{1}^{(4)}(0)}{\beta^{4} y^{4}} \frac{t^{4}}{4 !}\right)=\frac{2}{3}\left[\varphi_{1}(0)-\frac{\varepsilon^{2}}{\varepsilon_{e}^{2}} \varphi_{1}^{(4)}(0)\right] .
$$

In the integral with function $\varphi_{2}(z)$ in Eq. A.2 we perform twice integration by parts over $t$. We find

$$
\begin{aligned}
& J_{m 2}=-4 \operatorname{Re} \int_{0}^{1} d x(1-x)\left[\varphi_{2}(0)+\frac{1}{\beta^{2} y^{2}} \int_{0}^{\infty} d t e^{-t} \varphi_{2}^{(2)}(z)\right]=-2 \varphi_{2}(0)-4 \frac{\varepsilon}{\varepsilon_{e}} S, \\
& S=\operatorname{Im} \int_{0}^{1} \frac{(1-x)^{2} d x}{x} \int_{0}^{\infty} d t e^{-t} \varphi_{2}^{(2)}(z) .
\end{aligned}
$$


To calculate $S$ we choose the value $y_{0}$ in such way that $y_{0} \ll 1$ and $|\beta| y_{0} \gg 1$ and divide the integral over $x$ into two. The first one is

$$
\begin{aligned}
& S_{1}=\operatorname{Im} \int_{x_{0}}^{1} \frac{(1-x)^{2} d x}{x} \int_{0}^{\infty} d t e^{-t} \varphi_{2}^{(2)}(z) \simeq \operatorname{Im} \int_{x_{0}}^{1} \frac{(1-x)^{2} d x}{x} \frac{1}{\beta^{2} y^{2}} \varphi_{2}^{(4)}(0) \\
& \simeq-\frac{1}{|\beta|^{2}}\left(\frac{1}{x_{0}}-1+3 \ln x_{0}+\frac{5}{2}\right) \varphi_{2}^{(4)}(0) \simeq-\frac{1}{|\beta|^{2}}\left(\frac{1}{y_{0}^{2}}+6 \ln y_{0}+\frac{5}{2}\right) \varphi_{2}^{(4)}(0)
\end{aligned}
$$

In the second integral we pass to the variable $y$ :

$$
S_{2}=2 \operatorname{Im} \int_{0}^{y_{0}} \frac{d y}{y\left(1+y^{2}\right)^{3}} \int_{0}^{\infty} d t e^{-t} \varphi_{2}^{(2)}(z) \simeq 2 \operatorname{Im} \int_{0}^{y_{0}}\left(1-3 y^{2}\right) \frac{d y}{y} \int_{0}^{\infty} d t e^{-t} \varphi_{2}^{(2)}(z)
$$

Let us consider the integral

$$
\begin{aligned}
& S_{21}=2 \operatorname{Im} \int_{0}^{y_{0}} \frac{d y}{y} \int_{0}^{\infty} d t e^{-t} \varphi_{2}^{(2)}\left(\frac{t}{\beta y}\right)=\frac{1}{i} \int_{\beta^{*} y_{0}}^{\beta y_{0}} \frac{d y}{y} \int_{0}^{\infty} d t e^{-t} \varphi_{2}^{(2)}\left(\frac{t}{y}\right) \\
& \simeq \frac{1}{i} \int_{\beta^{*} y_{0}}^{\beta y_{0}} \frac{d y}{y}\left(\varphi_{2}^{(2)}(0)+\frac{1}{y^{2}} \varphi_{2}^{(4)}(0)\right)=\frac{1}{i}\left[\ln \frac{\beta}{\beta^{*}} \varphi_{2}^{(2)}(0)-\frac{1}{2}\left(\frac{1}{\beta^{2} y_{0}^{2}}-\frac{1}{\beta^{* 2} y_{0}^{2}}\right) \varphi_{2}^{(4)}(0)\right] \\
& =\frac{\pi}{2} \varphi_{2}^{(2)}(0)+\frac{1}{|\beta|^{2} y_{0}^{2}} \varphi_{2}^{(4)}(0) .
\end{aligned}
$$

The remaining integral in Eq. A.8 is

$$
\begin{aligned}
& S_{22}=-6 \operatorname{Im} \int_{0}^{y_{0}} y d y \int_{0}^{\infty} d t e^{-t} \varphi_{2}^{(2)}(z)=\frac{6}{|\beta|^{2}} \operatorname{Re} \int_{0}^{\beta y_{0}} \frac{d y}{y} \int_{0}^{\infty} d t e^{-t} \varphi_{2}^{(4)}\left(\frac{t}{y}\right) \\
& =\frac{6}{|\beta|^{2}} \operatorname{Re}\left[\ln \left(\beta y_{0}\right) \int_{0}^{\infty} d t e^{-t} \varphi_{2}^{(4)}\left(\frac{t}{\beta y_{0}}\right)+\int_{0}^{\beta y_{0}} \ln y d y \int_{0}^{\infty} \frac{t d t}{y^{2}} e^{-t} \varphi_{2}^{(5)}\left(\frac{t}{y}\right)\right] \\
& \simeq \frac{6}{|\beta|^{2}} \operatorname{Re}\left[\ln \left(\beta y_{0}\right) \varphi_{2}^{(4)}(0)+\int_{0}^{\infty} \ln y d y \int_{0}^{\infty} z e^{-z y} \varphi_{2}^{(5)}(z) d z\right] \\
& =\frac{6}{|\beta|^{2}} \operatorname{Re}\left[\ln \left(\beta y_{0}\right) \varphi_{2}^{(4)}(0)+\int_{0}^{\infty} d y \int_{0}^{\infty}(\ln y-\ln z) e^{-y} \varphi_{2}^{(5)}(z) d z\right] \\
& =\frac{6}{|\beta|^{2}} \operatorname{Re}\left[\left(\ln \left(\beta y_{0}\right)+C\right) \varphi_{2}^{(4)}(0)-\int_{0}^{\infty} \ln z \varphi_{2}^{(5)}(z) d z\right]
\end{aligned}
$$

Summing $S_{1}, S_{21}$ and $S_{22}$ we obtain for $S$

$$
S=\frac{\pi}{2} \varphi_{2}^{(2)}(0)+3 \frac{\varepsilon}{\varepsilon_{e}}\left[\left(\ln \frac{\varepsilon_{e}}{\varepsilon}+2 C-\frac{5}{6}\right) \varphi_{2}^{(4)}(0)-2 \int_{0}^{\infty} \ln z \varphi_{2}^{(5)}(z) d z\right] .
$$

Substituting Eq. (A.11) into $J_{m 2}$, adding with $J_{m 1}$ Eq.(A.5) and taking into account that

$$
\begin{aligned}
& \varphi_{1}(0)=\varphi_{1}^{(4)}(0)=\frac{1}{2}, \varphi_{2}(0)=-\frac{1}{3}, \varphi_{2}^{(2)}(0)=\frac{2}{15}, \varphi_{2}^{(4)}(0)=-\frac{16}{63}, \\
& \int_{0}^{\infty} \ln z \varphi_{2}^{(5)}(z) d z=-0.286,
\end{aligned}
$$


we obtain

$$
\begin{aligned}
& J_{m}=1-\frac{4 \pi}{15} \frac{\varepsilon}{\varepsilon_{e}}+\frac{64}{21} \frac{\varepsilon^{2}}{\varepsilon_{e}^{2}}\left(\ln \frac{\varepsilon_{e}}{\varepsilon}+2 C-\frac{5}{6}-\frac{7}{64}+\frac{63}{8} \int_{0}^{\infty} \ln z \varphi_{2}^{(5)}(z) d z\right)+O\left(\frac{\varepsilon^{3}}{\varepsilon_{e}^{3}}\right) \\
& =1-\frac{4 \pi}{15} \frac{\varepsilon}{\varepsilon_{e}}+\frac{64}{21} \frac{\varepsilon^{2}}{\varepsilon_{e}^{2}}\left(\ln \frac{\varepsilon_{e}}{\varepsilon}-2.040\right)+O\left(\frac{\varepsilon^{3}}{\varepsilon_{e}^{3}}\right) .
\end{aligned}
$$

Here the first two terms agree with Eq.(3.9) in [10].

\section{B Appendix}

Here we calculate the function $J_{v}$ in Eq.A.1. defined in Eq.A.3. In the last expression one can expand the function $l_{1}(z, t)$ up to terms $\propto z^{4}$. We find

$$
\begin{aligned}
& J_{v 1} \simeq\left(\frac{\varepsilon}{\varepsilon_{e}}\right)^{2} \int_{0}^{1}(1-x)^{2} d x \int_{0}^{\infty} d t e^{-t} \frac{t^{4}}{4 !}\left(a_{1}^{(4)}(0)(\ln t+C)+b_{1}^{(4)}(0)\right) \\
& =\frac{1}{3}\left(\frac{\varepsilon}{\varepsilon_{e}}\right)^{2}\left[(\psi(5)-\psi(1)) a_{1}^{(4)}(0)+b_{1}^{(4)}(0)\right]=\frac{1}{3}\left(\frac{\varepsilon}{\varepsilon_{e}}\right)^{2}\left[\frac{25}{12} a_{1}^{(4)}(0)+b_{1}^{(4)}(0)\right],
\end{aligned}
$$

where the function $\psi(x)$ is defined in Eq.(2.2). The integral with the function $l_{2}(z, t)$ is

$$
J_{v 2}=-4 \operatorname{Re} \int_{0}^{1}(1-x) d x \int_{0}^{\infty} d t e^{-t}\left[a_{2}(z)(\ln t+C)+b_{2}(z)\right] .
$$

The term with $b_{2}(z)$ can be calculated as the term $J_{m 2}$ in the Appendix A. Substituting $\varphi_{2}(z) \rightarrow b_{2}(z)$ we have

$$
\begin{aligned}
& J_{v 2}^{(b)}=-2 b_{2}(0)-2 \pi \frac{\varepsilon}{\varepsilon_{e}} b_{2}^{(2)}(0) \\
& -12\left(\frac{\varepsilon}{\varepsilon_{e}}\right)^{2}\left[\left(\ln \frac{\varepsilon_{e}}{\varepsilon}+2 C-\frac{5}{6}\right) b_{2}^{(4)}(0)-2 \int_{0}^{\infty} \ln z b_{2}^{(5)}(z) d z\right] .
\end{aligned}
$$

Now we turn over to the term with $a_{2}(z)$ in Eq.(B.2)

$$
\begin{aligned}
& T=\operatorname{Re} \int_{0}^{1}(1-x) d x \int_{0}^{\infty} d t e^{-t} a_{2}(z)(\ln t+C) \\
& =\operatorname{Re} \int_{0}^{1}(1-x) d x \int_{0}^{\infty} d t e^{-t}\left(a_{2}(z)-a_{2}(0)\right)(\ln t+C) .
\end{aligned}
$$

As in previous Appendix we divide the integral over $x$ into two: $0 \leq x \leq x_{0}$ and $x_{0} \leq x \leq 1$. We have (see Eqs.(B.1) and (A.7)

$$
\begin{aligned}
& T_{1}=\operatorname{Re} \int_{x_{0}}^{1}(1-x) d x \int_{0}^{\infty} d t e^{-t}\left(a_{2}(z)-a_{2}(0)\right)(\ln t+C) \\
& =-\frac{1}{|\beta|^{4}} \int_{x_{0}}^{1} \frac{(1-x)^{3}}{x^{2}} d x \frac{25}{12} a_{1}^{(4)}(0) \simeq-\frac{25}{12|\beta|^{4}}\left(\frac{1}{y_{0}^{2}}+6 \ln y_{0}+\frac{5}{2}\right) a_{2}^{(4)}(0) .
\end{aligned}
$$


In the second integral we perform twice integration by parts over $t$ and pass to the variable $y$. We find

$$
\begin{aligned}
& T_{2}=\frac{2}{|\beta|^{2}} \operatorname{Im} \int_{0}^{y_{0}} \frac{d y}{y\left(1+y^{2}\right)^{3}} \int_{0}^{\infty} d t e^{-t}\left[a_{2}^{(2)}(z)(\ln t+C)+d(z)\right] \\
& d(z)=\frac{2}{z} a_{2}^{(1)}(z)-\frac{1}{z^{2}}\left(a_{2}(z)-a_{2}(0)\right) .
\end{aligned}
$$

We proceed as above (see Eq.(A.8) ) and expand $\left(1+y^{2}\right)^{3} \simeq 1-3 y^{2}$, then

$$
\begin{aligned}
& T_{21}=\frac{2}{|\beta|^{2}} \operatorname{Im} \int_{0}^{\beta y_{0}} \frac{d y}{y} \int_{0}^{\infty} d t e^{-t}\left[a_{2}^{(2)}\left(\frac{t}{y}\right)(\ln t+C)+d\left(\frac{t}{y}\right)\right] \\
& =\frac{1}{|\beta|^{2}} \frac{1}{i} \int_{\beta^{*} y_{0}}^{\beta y_{0}} \frac{d y}{y} \int_{0}^{\infty} d t e^{-t}\left[a_{2}^{(2)}\left(\frac{t}{y}\right)(\ln t+C)+d\left(\frac{t}{y}\right)\right] \\
& \simeq \frac{1}{|\beta|^{2}}\left[\frac{\pi}{2} d(0)+\frac{1}{|\beta|^{2} y_{0}^{2}}\left(\frac{3}{2} a_{2}^{(4)}(0)+d^{(2)}(0)\right)\right] \\
& =\frac{1}{|\beta|^{2}}\left[\frac{3 \pi}{4} a_{2}^{(2)}(0)+\frac{25}{12|\beta|^{2} y_{0}^{2}} a_{2}^{(4)}(0)\right] .
\end{aligned}
$$

and

$$
\begin{aligned}
& T_{22}=-\frac{6}{|\beta|^{2}} \operatorname{Im} \int_{0}^{y_{0}} y d y \int_{0}^{\infty} d t e^{-t}\left[a_{2}^{(2)}(z)(\ln t+C)+d\left(\frac{t}{y}\right)\right] \\
& =-\frac{6}{|\beta|^{2}} \operatorname{Im} \int_{0}^{y_{0}} y d y \int_{0}^{\infty} d t e^{-t}\left[\left(a_{2}^{(2)}(z)-a_{2}^{(2)}(0)\right)(\ln t+C)+d\left(\frac{t}{y}\right)\right] .
\end{aligned}
$$

Integrating by parts over t two times we obtain

$$
\begin{aligned}
& T_{22}=\frac{6}{|\beta|^{4}} \operatorname{Re} \int_{0}^{\beta y_{0}} \frac{d y}{y} \int_{0}^{\infty} d t e^{-t}\left[a_{2}^{(4)}\left(\frac{t}{y}\right)(\ln t+C)+g\left(\frac{t}{y}\right)\right] \\
& g(z)=\frac{2}{z} a_{2}^{(3)}(z)-\frac{1}{z^{2}}\left(a_{2}^{(2)}(z)-a_{2}^{(2)}(0)\right)+d^{(2)}(z) .
\end{aligned}
$$

The term with the function $g(z)$ can be calculated in the same manner as in Eq. (A.10) $\left(\varphi_{2}^{(4)}(z) \rightarrow g(z)\right)$

$$
T_{22}^{(g)}=\frac{6}{|\beta|^{4}} \operatorname{Re}\left[\left(\ln \left(\beta y_{0}\right)+C\right) g(0)-\int_{0}^{\infty} \ln z g^{\prime}(z) d z\right]
$$

Now we have to calculate the integral containing $\ln t+C$

$$
\begin{aligned}
& T_{22}^{(l)}=\frac{6}{|\beta|^{4}} \operatorname{Re} \int_{0}^{\beta y_{0}} \frac{d y}{y} \int_{0}^{\infty} e^{-t} a_{2}^{(4)}\left(\frac{t}{y}\right)(\ln t+C) d t \\
& \simeq \frac{6}{|\beta|^{4}} \int_{0}^{\infty} \ln y d y \int_{0}^{\infty} z e^{-z y}(\ln y+\ln z+C) a_{2}^{(5)}(z) d z
\end{aligned}
$$




$$
\begin{aligned}
& =\frac{6}{|\beta|^{4}} \int_{0}^{\infty} d y \int_{0}^{\infty}(\ln y-\ln z)(\ln y+C) e^{-y} a_{2}^{(5)}(z) d z \\
& =-\frac{6}{|\beta|^{4}} a_{2}^{(4)}(0) \int_{0}^{\infty} e^{-y}\left(\ln ^{2} y-C^{2}\right) d y=-\frac{\pi^{2}}{|\beta|^{4}} a_{2}^{(4)}(0)
\end{aligned}
$$

Taking into account that $g(0)=\frac{25}{12} a_{2}^{(4)}(0)$ and summing $T_{1}$ Eq.(B.5),$T_{21}$ Eq.(B.7), $T_{22}^{(g)}$ Eq.(B.10), $T_{22}^{(l)}$ Eq.(B.11) we find for $T$ Eq.(B.4)

$$
\begin{aligned}
& T=\frac{3 \pi}{4} \frac{\varepsilon}{\varepsilon_{e}} a_{2}^{(2)}(0)+\frac{\varepsilon^{2}}{\varepsilon_{e}^{2}}\left[\frac{25}{4}\left(\ln \frac{\varepsilon_{e}}{\varepsilon}+2 C-\frac{5}{6}-\frac{4}{25} \pi^{2}\right) a_{2}^{(4)}(0)-6 \int_{0}^{\infty} \ln z g^{\prime}(z) d z\right] ; \\
& g(z)=\frac{4}{z} a_{2}^{(3)}(z)-\frac{1}{z^{2}}\left(6 a_{2}^{(2)}(z)-a_{2}^{(2)}(0)\right)+\frac{8}{z^{3}} a_{2}^{(1)}(z)-\frac{6}{z^{4}}\left(a_{2}(z)-a_{2}(0)\right) .
\end{aligned}
$$

Substituting the obtained expression for $T$ Eq. (B.12) and $J_{v 2}^{(b)}$ Eq. (B.3) into $J_{v 2}$, adding to $J_{v 1}$ and taking into account that

$$
\begin{aligned}
& a_{1}^{(4)}(0)=\frac{1}{30}, b_{1}^{(4)}(0)=\frac{1223}{450}, a_{2}^{(2)}(0)=-\frac{4}{15}, a_{2}^{(4)}(0)=\frac{16}{21}, \\
& b_{2}(0)=-\frac{1}{18}, b_{2}^{(2)}(0)=-\frac{1}{225}, b_{2}^{(4)}(0)=\frac{106}{735},
\end{aligned}
$$

and numerical value of integrals

$$
\int_{0}^{\infty} \ln z b_{2}^{(5)}(z) d z \simeq 0.250, \quad \int_{0}^{\infty} \ln z g^{\prime}(z) d z \simeq 0.967
$$

we obtain

$$
J_{v}=\frac{1}{9}+\frac{182 \pi}{225} \frac{\varepsilon}{\varepsilon_{e}}-\frac{15272}{735} \frac{\varepsilon^{2}}{\varepsilon_{e}^{2}}\left(\ln \frac{\varepsilon_{e}}{\varepsilon}-2.577\right)+O\left(\frac{\varepsilon^{3}}{\varepsilon_{e}^{3}}\right) .
$$

\section{Appendix}

Here we consider the asymptotic behavior of the total probability of radiation $W$ in the region $\varepsilon \ll \varepsilon_{e}$ to within terms $\sim \varepsilon / \varepsilon_{e}$ inclusively. According with Eqs. A.1 - A.4 we present the total probability in form

$$
W=\frac{1}{L_{r a d}^{0}}\left(W_{m}+\frac{1}{L_{1}} W_{v}\right)
$$

where

$$
\begin{aligned}
& W_{m}=2 \operatorname{Re} \int_{0}^{1} \frac{d x}{x} \int_{0}^{\infty} d t e^{-t}\left[x^{2} \varphi_{1}(z)-2(1-x) \varphi_{2}(z)\right] \\
& W_{v}=-\operatorname{Re} \int_{0}^{1} \frac{d x}{x} \int_{0}^{\infty} d t e^{-t}\left[x^{2} l_{1}(z, t)+4(1-x) l_{2}(z, t)\right]
\end{aligned}
$$


The function entering in Eq. (C.2) are defined in Eqs. A.3)- A.4 .

The term with $\varphi_{1}(z)$ in the integrand of $W_{m}$ to within mentioned accuracy is

$$
W_{m 1}=2 \int_{0}^{1} x d x \int_{0}^{\infty} e^{-t} d t \varphi_{1}(0)
$$

In the integral with $\varphi_{2}(z)$ we divide the integral over $x$ into two

$$
W_{m 2}^{(1)}=-4 \operatorname{Re} \int_{x_{0}}^{1} \frac{d x}{x}(1-x) \int_{0}^{\infty} d t e^{-t} \varphi_{2}(z) \simeq 4\left(\ln x_{0}+1\right) \varphi_{2}(0),
$$

and

$$
\begin{aligned}
& W_{m 2}^{(2)}=-8 \operatorname{Re} \int_{0}^{y_{0}} \frac{d y}{y\left(1+y^{2}\right)^{2}} \int_{0}^{\infty} d t e^{-t} \varphi_{2}(z) \simeq-8 \operatorname{Re} \int_{0}^{\beta y_{0}} \frac{d y}{y} \int_{0}^{\infty} d t e^{-t} \varphi_{2}\left(\frac{t}{y}\right) \\
& +16 \operatorname{Re} \int_{0}^{y_{0}} y d y \int_{0}^{\infty} d t e^{-t} \varphi_{2}\left(\frac{t}{\beta y}\right)=W_{m 21}^{(2)}+W_{m 22}^{(2)}
\end{aligned}
$$

Integration of $W_{m 21}^{(2)}$ coincides with calculation in Eq.(A.10) with substitution $\varphi_{2}^{(4)}\left(\frac{t}{y}\right) \rightarrow \varphi_{2}\left(\frac{t}{y}\right)$. We have

$$
\begin{aligned}
& W_{m 21}^{(2)}=-8 \operatorname{Re} \int_{0}^{\beta y_{0}} \frac{d y}{y} \int_{0}^{\infty} d t e^{-t} \varphi_{2}\left(\frac{t}{y}\right) \\
& \simeq-4\left[\left(\ln \frac{\varepsilon_{e}}{\varepsilon}+2 C+\ln y_{0}^{2}\right) \varphi_{2}(0)-2 \int_{0}^{\infty} \ln z \varphi_{2}^{(1)}(z) d z\right]
\end{aligned}
$$

Integrating by parts twice over $t$ in integral $W_{m 22}^{(2)}$ Eq.(C.5) we find (see Eq.(A.9)

$$
W_{m 22}^{(2)}=16 \frac{\varepsilon}{\varepsilon_{e}} \operatorname{Im} \int_{0}^{\beta y_{0}} \frac{d y}{y} \int_{0}^{\infty} d t e^{-t} \varphi_{2}^{(2)}\left(\frac{t}{y}\right) \simeq 4 \pi \frac{\varepsilon}{\varepsilon_{e}} \varphi_{2}^{(2)}(0) .
$$

Summing $W_{m 2}^{(1)}$ Eq.(C.4), $W_{m 21}^{(2)}$ Eq.(C.6) and $W_{m 22}^{(2)}$ Eq.(C.7) and taking into account Eq.(A.12)) we have

$$
\begin{aligned}
& W_{m}=-4 \varphi_{2}(0)\left[\ln \frac{\varepsilon_{e}}{\varepsilon}+2 C-1-\frac{\varphi_{1}(0)}{4 \varphi_{2}(0)}-\frac{2}{\varphi_{2}(0)} \int_{0}^{\infty} \ln z \varphi_{2}^{(1)}(z) d z\right]+ \\
& 4 \pi \frac{\varepsilon}{\varepsilon_{e}} \varphi_{2}^{(2)}(0)=\frac{4}{3}\left[\ln \frac{\varepsilon_{e}}{\varepsilon}+2 C-\frac{5}{8}+12 \int_{0}^{\infty} \ln z\left(\frac{1}{z^{3}}-\frac{\cosh z}{\sinh ^{3} z}\right) d z\right]+\frac{8 \pi}{15} \frac{\varepsilon}{\varepsilon_{e}} \\
& =\frac{4}{3}\left(\ln \frac{\varepsilon_{e}}{\varepsilon}+1.959\right)+\frac{8 \pi}{15} \frac{\varepsilon}{\varepsilon_{e}}
\end{aligned}
$$

The first term in Eq.(C.8) ) is similar to Eq.(2.21) in [9]. 
We will consider now the probability $W_{v}$ in Eq.(C.2) $)$. The term with $l_{1}(z, t)$ gives no contribution into two first terms of expansion over $\frac{\varepsilon}{\varepsilon_{e}}$.

$$
W_{v 2}=-4 \operatorname{Re} \int_{0}^{1}(1-x) \frac{d x}{x} \int_{0}^{\infty} d t e^{-t}\left[a_{2}(z)(\ln t+C)+b_{2}(z)\right]=W_{v 2}^{(a)}+W_{v 2}^{(b)}
$$

The calculation of the term $W_{v 2}^{(b)}$ (with $b_{2}(z)$ ) is analogous to the calculation above (with substitution $\varphi_{2}(z) \rightarrow b_{2}(z)$ )

$$
W_{v 2}^{(b)}=-4 b_{2}(0)\left(\ln \frac{\varepsilon_{e}}{\varepsilon}+2 C-1\right)+8 \int_{0}^{\infty} \ln z b_{2}^{(1)}(z) d z+4 \pi \frac{\varepsilon}{\varepsilon_{e}} b_{2}^{(2)}(0) .
$$

For calculation of term $W_{v 2}^{(a)}$ (with $\left.a_{2}(z)\right)$ it is convenient to use the variable y

$$
\begin{aligned}
& W_{v 2}^{(a)}=W_{v 21}^{(a)}+W_{v 22}^{(a)} ; \\
& W_{v 21}^{(a)}=-8 \int_{0}^{\infty} \frac{d y}{y} \int_{0}^{\infty} d t e^{-t}\left(a_{2}\left(\frac{t}{y}\right)-a_{2}\left(\frac{1}{y}\right)\right)(\ln t+C), \\
& W_{v 22}^{(a)}=8 \operatorname{Re} \int_{0}^{\infty} \frac{d y}{y}\left(1-\frac{1}{\left(1+y^{2}\right)^{2}}\right) \int_{0}^{\infty} d t e^{-t}\left(a_{2}(z)-a_{2}(0)\right)(\ln t+C) .
\end{aligned}
$$

The integral over $y$ in $W_{v 21}^{(a)}$ is the integral Frullani

$$
\int_{0}^{\infty}\left(a_{2}\left(\frac{t}{y}\right)-a_{2}\left(\frac{1}{y}\right)\right) \frac{d y}{y}=-a_{2}(0) \ln t .
$$

Using the last result in Eq.(B.11) we have

$$
W_{v 21}^{(a)}=\frac{4}{3} \pi^{2} a_{2}(0)
$$

In the expression for $W_{v 22}^{(a)}$ we divide the integration interval into two parts:

$$
\begin{aligned}
& W_{v 22}^{(a 1)}=8 \operatorname{Re} \int_{y_{0}}^{\infty} \frac{d y}{y}\left(1-\frac{1}{\left(1+y^{2}\right)^{2}}\right) \int_{0}^{\infty} e^{-t}\left(a_{2}^{(2)}(0) \frac{t^{2}}{2 ! \beta^{2} y^{2}}+\ldots\right)(\ln t+C) d t=0 \\
& W_{v 22}^{(a 2)} \simeq 16 \operatorname{Re} \int_{0}^{y_{0}} y d y \int_{0}^{\infty} e^{-t}\left(a_{2}(z)-a_{2}(0)\right)(\ln t+C) d t \\
& =16 \operatorname{Im} \int_{0}^{\beta y_{0}} \frac{d y}{y} \int_{0}^{\infty} e^{-t}\left[a_{2}^{(2)}\left(\frac{t}{y}\right)(\ln t+C)+d\left(\frac{t}{y}\right)\right] d t
\end{aligned}
$$

The function $d(z)$ is defined in Eq.(B.6) , the further calculation can be done as in Eq. B.7

$$
W_{v 22}^{(a 2)} \simeq 4 \pi \frac{\varepsilon}{\varepsilon_{e}} d(0)=6 \pi \frac{\varepsilon}{\varepsilon_{e}} a_{2}^{(2)}(0) .
$$


Summing $W_{v 2}^{(b)}$ Eq. (C.10), $W_{v 21}^{(a)}$ Eq. (C.13) and $W_{v 21}^{(a)}$ Eq.C.15) we obtain the following expression for $W_{v}$ Eq. (C.1)

$$
W_{v}=-4 b_{2}(0)\left(\ln \frac{\varepsilon_{e}}{\varepsilon}+2 C-1\right)+8 \int_{0}^{\infty} \ln z b_{2}^{(1)}(z) d z+\frac{4 \pi^{2}}{3}+2 \pi \frac{\varepsilon}{\varepsilon_{e}}\left(3 a_{2}^{(2)}(0)+2 b_{2}^{(2)}(0)\right) \text {. }
$$

Taking into account that

$$
\int_{0}^{\infty} \ln z b_{2}^{(1)}(z) d z=0.0431
$$

and using Eq. (B.13) we obtain $\left(a_{2}(0)=1 / 3\right)$

$$
W_{v}=\frac{2}{9} \ln \frac{\varepsilon_{e}}{\varepsilon}+4.7655-\frac{364 \pi}{225} \frac{\varepsilon}{\varepsilon_{e}}+O\left(\frac{\varepsilon^{2}}{\varepsilon_{e}^{2}}\right) .
$$

\section{Appendix}

Here we discuss a structure of corrections to the total intensity of radiation at $\varepsilon \ll \varepsilon_{e}$ which is not included in the expansion over $1 / L_{c}$ contained in Eqs.(2.1)(2.4). We will show that these corrections, which are contained terms $\propto 1 / L_{1}^{2}$ and higher degrees of $1 / L_{1}$, are beginning from terms of the order $\varepsilon / \varepsilon_{e}$. We will use the general expression Eq.(2.12) of [7] before decomposition over $1 / L$ :

$$
\frac{d I}{d x}=\frac{2 \alpha m^{2} x}{1-x} \operatorname{Im}\left\langle 0\left|r_{1}\left(G^{-1}-G_{0}^{-1}\right)+r_{2} \mathbf{p}\left(G^{-1}-G_{0}^{-1}\right) \mathbf{p}\right| 0\right\rangle,
$$

where

$$
G=\mathbf{p}^{2}+1-i V(\varrho), G_{0}=\mathbf{p}^{2}+1, V(\varrho)=\frac{\varepsilon}{4 \varepsilon_{e}} \frac{1-x}{x} \varrho^{2}\left(L_{1}-\ln \frac{\varrho^{2}}{4}-2 C\right),
$$

here the quantities $r_{1}, r_{2}$ are defined in Eq.(2.2) and $\varepsilon_{e}$ and $L_{1}$ are defined in Eq.(2.4). In the region $1 \geq x \gg \varepsilon / \varepsilon_{e}$ we expand the combination entering in Eq.(D.1) over degrees of $V$

$$
G^{-1}-G_{0}^{-1}=G_{0}^{-1} i V G_{0}^{-1}+G_{0}^{-1} i V G_{0}^{-1} i V G_{0}^{-1}+G_{0}^{-1} i V G_{0}^{-1} i V G_{0}^{-1} i V G_{0}^{-1}+\ldots
$$

Substituting Eq. (D.3) into Eq.(D.1) one can verify that the terms of the order $1 / L_{1}^{2}$ and higher degrees of $1 / L_{1}$ are appeared starting from the third term of decomposition Eq.(D.3). Their relative contribution is of the order $\varepsilon^{2} / \varepsilon_{e}^{2}$. Taking into account that

$$
G_{0}^{-1}=i \int_{0}^{\infty} e^{-i t} e^{-i \mathbf{p}^{2} t} d t, \quad<\varrho_{1}\left|e^{-i \mathbf{p}^{2} t}\right| \varrho_{2}>=\frac{1}{4 \pi i t} e^{\frac{i}{4 t}\left(\boldsymbol{\varrho}_{1}-\boldsymbol{\varrho}_{2}\right)^{2}}
$$

we obtain

$$
<\varrho_{1}\left|G_{0}^{-1}\right| \varrho_{2}>=\frac{1}{4 \pi} \int_{0}^{\infty} \exp \left[-i t+\frac{i}{4 t}\left(\boldsymbol{\varrho}_{1}-\boldsymbol{\varrho}_{2}\right)^{2}\right] \frac{d t}{t}=\frac{1}{2 \pi} K_{0}\left(\left|\varrho_{1}-\varrho_{2}\right|\right)
$$


where $K_{0}(z)$ is the modified Bessel function (Mac-Donald's function). Substituting this result into Eq.(D.3) and then into Eq.(D.1) we get

$$
\begin{aligned}
& \frac{d I}{d x}=\frac{\alpha m^{2} x}{\pi(1-x)} \int_{0}^{\infty}\left[r_{1} K_{0}^{2}(\varrho)+r_{2} K_{1}^{2}(\varrho)\right] V(\varrho) \varrho d \varrho \\
& =\frac{\alpha m^{2}}{4 \pi} \frac{\varepsilon}{\varepsilon_{e}}\left[\frac{r_{1}+2 r_{2}}{3} L_{1}+\frac{r_{2}-r_{1}}{9}\right], \quad I^{(0)}=\frac{\alpha m^{2}}{4 \pi} \frac{\varepsilon}{\varepsilon_{e}}\left(L_{1}+\frac{1}{9}\right) .
\end{aligned}
$$

Obtained here result for $I^{(0)}$ agrees with Bethe-Maximon formula.

In the region $x \sim \varepsilon / \varepsilon_{e}$ the potential $V \sim 1$ and decomposition Eq.(D.3) is inapplicable. However in this region

$$
d I \sim \alpha m^{2} \frac{x d x}{1-x} \sim \alpha m^{2}\left(\frac{\varepsilon}{\varepsilon_{e}}\right)^{2}
$$

and the relative contribution of this region $\sim \varepsilon / \varepsilon_{e}$. 


\section{References}

[1] V.N.Baier, V.M.Katkov and V.M.Strakhovenko, Electromagnetic Processes at High Energies in Oriented Single Crystals, World Scientific Publishing Co, Singapore, 1998.

[2] L. D. Landau and I. Ya. Pomeranchuk, Dokl.Akad.Nauk SSSR 92 (1953) 535, 735. See The Collected Paper of L. D. Landau (Pergamon Press, New York 1965) for an English translation.

[3] A. B. Migdal, Phys. Rev. 103 (1956) 1811.

[4] P. L. Anthony, R. Becker-Szendy, P. E. Bosted, et al, Phys.Rev.D 56 (1997) 1373.

[5] H. D. Hansen, U. I. Uggerhoj, C.C.Biino et al, Phys.Rev.Lett. 91 (2003) 014801.

[6] H. D. Hansen, U. I. Uggerhoj, C.C.Biino et al, Phys.Rev.D 69 (2004) 032001.

[7] V. N. Baier and V. M. Katkov Phys.Rev. D 57 (1998) 3146.

[8] V. N. Baier and V. M. Katkov, "The Landau-Pomeranchuk-Migdal effect in a thin target", in Quantum Aspects of Beam Physics, ed.P. Chen, World Scientific PC, Singapore, 1998, p.525.

[9] V. N. Baier and V. M. Katkov, Phys.Rev.D 59 (1999) 056003.

[10] V. N. Baier and V. M. Katkov, Phys.Rev. D 62 (2000) 036008.

[11] B. G. Zakharov, Pis'ma v ZhETF 63 (1996) 906.

[12] R. Baier, Yu. L. Dokshitzer, A. H. Mueller, S. Peigne, and D. Schiff, Nucl. Phys. B478 (1996) 577.

[13] V. N. Baier and V. M. Katkov, "Concept of formation length in radiation theory" hep-ph/0309211, 2003. 


\section{Figure captions}

- Fig.1 The energy loss spectrum $\frac{d \varepsilon}{d \omega}$ in units $\frac{1}{L_{\text {rad }}^{0}}$ in iridium target with thickness $l=0.128 \mathrm{~mm}\left(l / L_{\text {rad }}^{B M}=4.36 \%\right)$ for the initial electrons energy is $\varepsilon=286.6 \mathrm{GeV}$. The Coulomb corrections are included.

- Curve 1 is the Bethe-Maximon spectrum,

- curve 2 is the contribution of the main term describing LPM effect;

- curve 3 is the correction term;

- curve 4 is the sum of two previous contributions;

- curve $\mathrm{T}$ is the final theory prediction with regard for the reduction factor (the multiphoton effects).

Experimental data from [5], 6].

- Fig.2 The same as in Fig.1 but for the initial electrons energy $\varepsilon=206.7 \mathrm{GeV}$.

- Fig.3 The same as in Fig.1 but for tantalum target with thickness $\left(l / L_{\text {rad }}^{B M}=\right.$ $4.45 \%)$.

- Fig.4 The reduction factor for iridium target with thickness $l=0.128 \mathrm{~mm}$ $\left(4.36 \% L_{r a d}\right)$ versus $x=\omega / \varepsilon$. The curves $1,2,3$ are for energies $149 \mathrm{GeV}$, $207 \mathrm{GeV}$ and $287 \mathrm{GeV}$ correspondingly.

- Fig.5 The relative energy losses of electron per unit time including the contribution of the correction term $(I(\varepsilon) / \varepsilon) L_{\text {rad }}^{B M}=L_{\text {rad }}^{B M} / L_{\text {rad }}$ in iridium (curve 1) and in lead (curve 2) vs the initial energy of electron. 


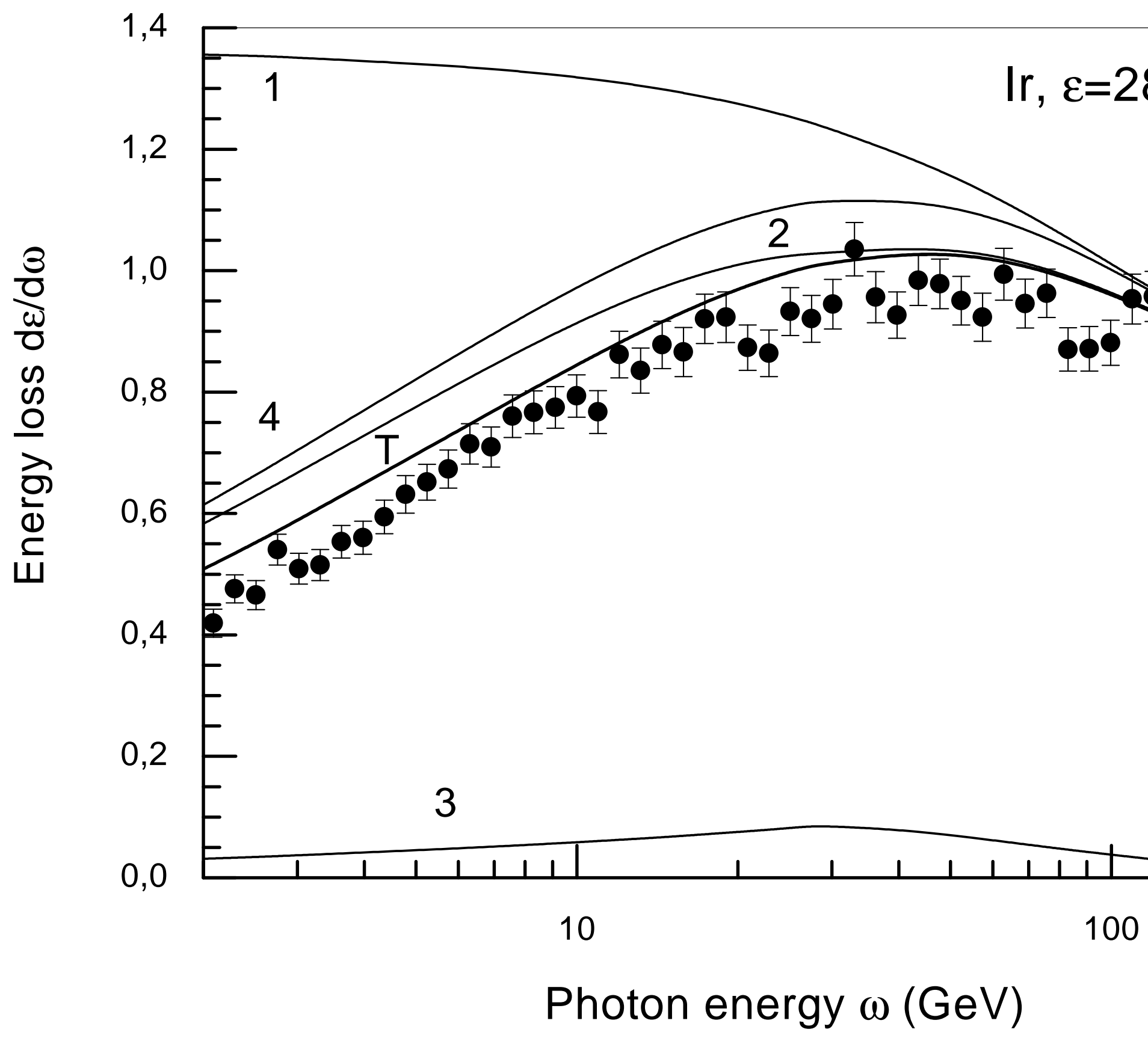




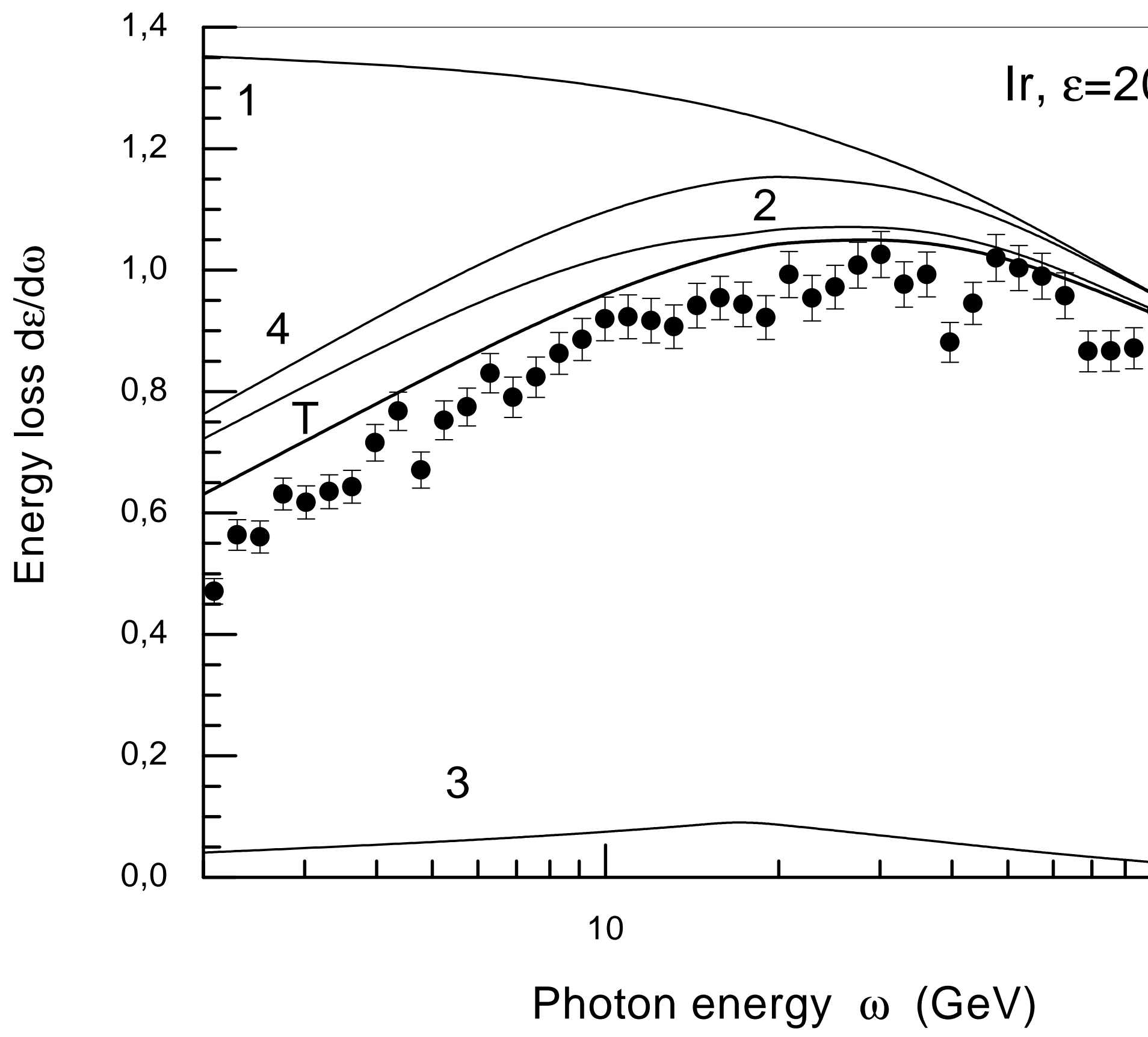




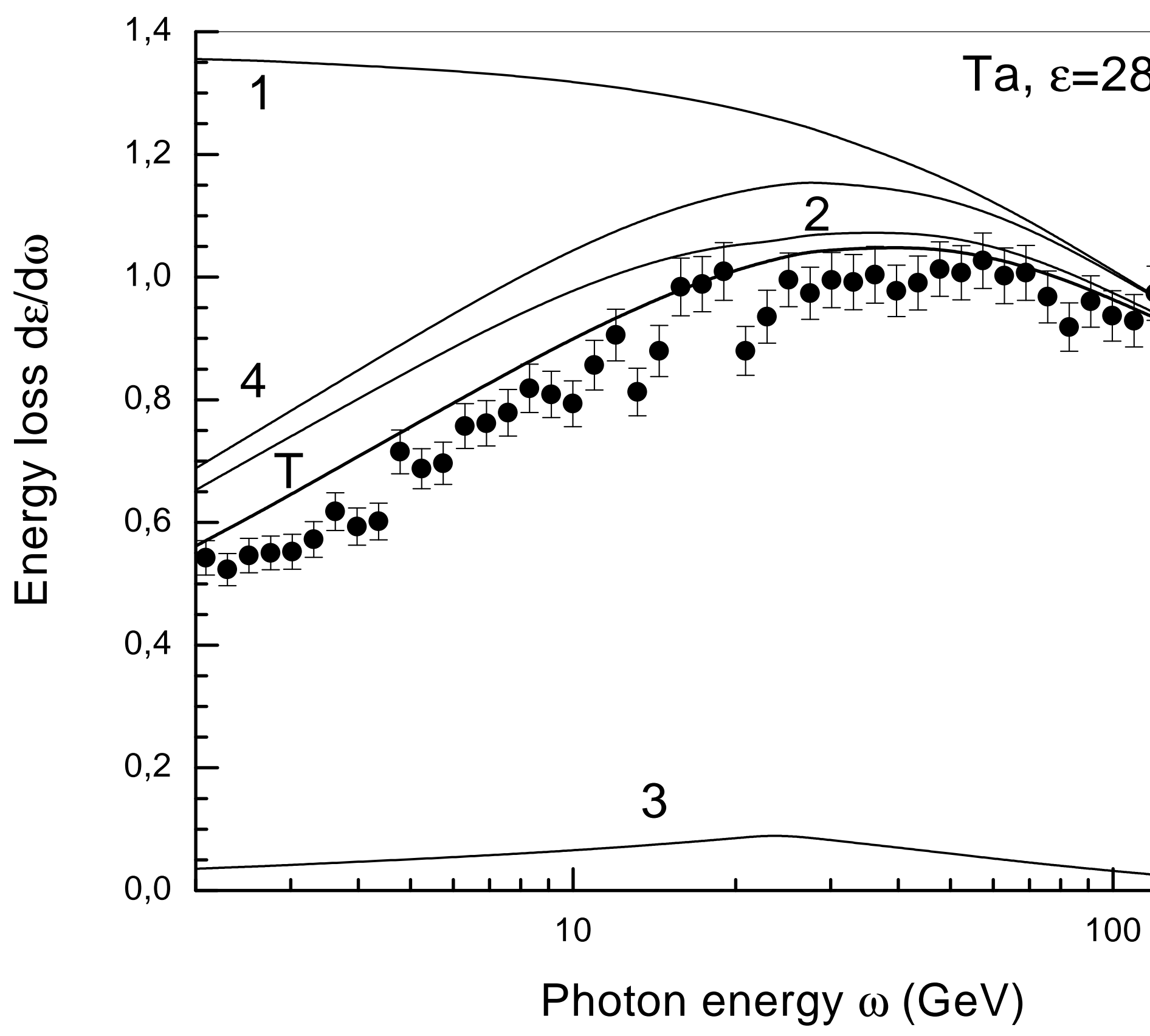




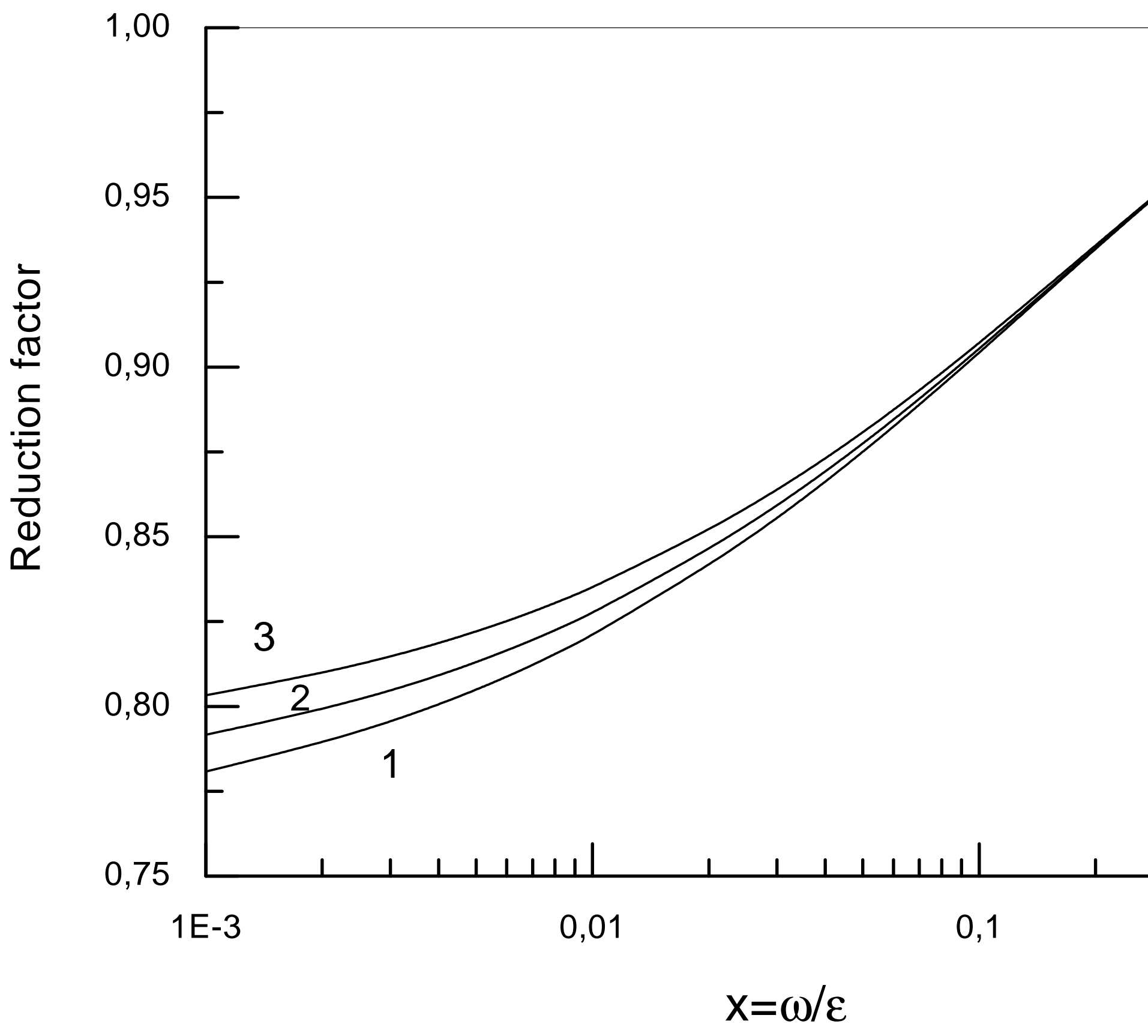




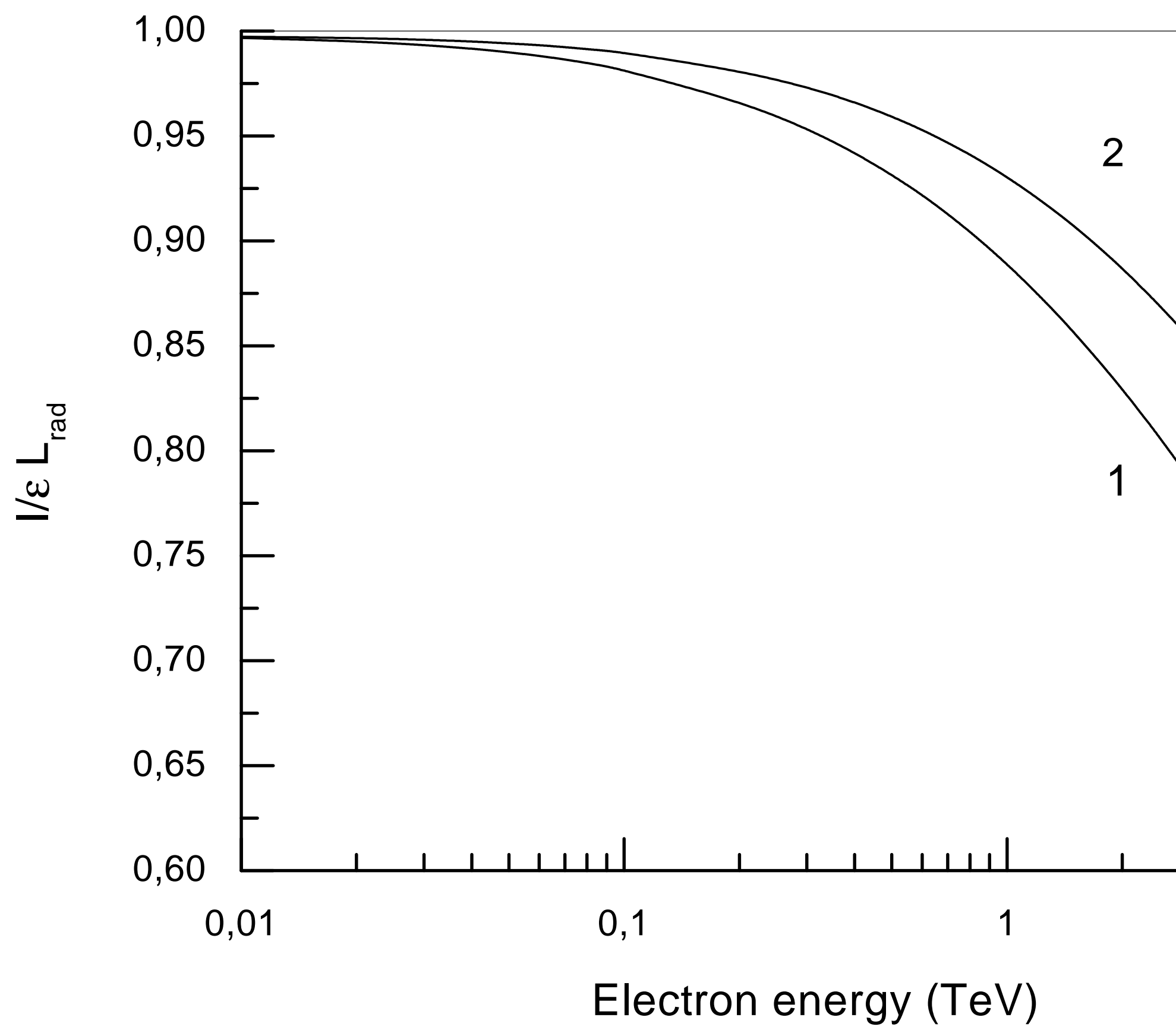

